\title{
Cryptanalysis of the Chang-Wu-Chen key distribution system
}

\author{
Mike Burmester \\ RH - University of London, Egham, Surrey TW20 OEX, U.K.
}

\begin{abstract}
Chang-Wu-Chen presented at Auscrypt 92 a conference key distribution system based on public keys. We show that this scheme is insecure and discuss ways to fix it.
\end{abstract}

\section{The CWC key distribution system}

This system [3] uses a discrete logarithm setting with prime modulus $p$ and primitive element $g$. Each party $U_{j}, j=1,2, \ldots, n$, has a secret key $x_{j} \in Z_{p-1}$ and a public key $y_{j}=g^{x_{j}}$ modp. A chairperson $U_{0}$ with secret key $x_{0} \in Z_{p-1}$ and public key $y_{0}=g^{x_{0}} \bmod p$ picks a random $r \in Z_{p-1}$ and computes $Y=$ $\prod_{i=1}^{n}\left(y_{i}\right)^{r} \bmod p$. The conference key is $k \equiv Y^{-1}(\bmod p)$. Then the chairperson sends each $U_{j}: c_{1}=g^{r} \bmod p, c_{2}=\left(y_{0}\right)^{k} \bmod p$, and $Y_{j} \equiv Y /\left(y_{j}\right)^{r}(\bmod p)$. The parties $U_{j}$ can easily compute $k$, since $k \equiv\left(Y_{j} \cdot\left(c_{1}\right)^{x_{j}}\right)^{-1}(\bmod p)$. To validate $k$, $U_{j}$ checks that $c_{2}=\left(y_{0}\right)^{k} \bmod p$.

\section{A cryptanalytic attack}

We have

$$
\prod_{i=1}^{n} Y_{i} \equiv \prod_{i=1}^{n}\left(Y /\left(y_{i}\right)^{r}\right) \equiv Y^{n-1} \equiv k^{1-n}(\bmod p) .
$$

So a passive eavesdropper can easily compute $k^{n-1} \bmod p$. Since it is feasible $[7,1]$ to compute $(n-1)$-th roots in $Z_{p}$, the eavesdropper will succeed in finding the key $k$ (with non-negligible probability) when $n \geq 2 .^{1}$

\section{Authentication}

In a key distribution system each party should know with whom it is exchanging the key. With the CWC system it is clear that the chairperson can substitute some of the parties $U_{1}, U_{2}, \ldots, U_{n}$ without the others finding this out from the key distribution system. So it is essential that the parties trust the chairperson. However the chairperson $U_{0}$ is not authenticated. Indeed the secret key $x_{0}$ of $U_{0}$ is not needed to compute either the validator $c_{2}$, or any of $c_{1}, Y_{j}$ and the key $k$. So anyone can easily masquerade as $U_{0}$ (by substituting its messages).

\footnotetext{
1 Edward Zuk from Telecom Research Laboratories, Australia, has found this attack independently. This was pointed out to me by Jennifer Seberry.
} 


\section{Fixing the system}

\subsection{Using a prime modulus}

We get some protection from the attack in Section 2 by replacing $Y$ and $Y_{j}$ by $\tilde{Y} \equiv\left(y_{0}\right)^{r(n-1)} \cdot \prod_{i=1}^{n}\left(y_{i}\right)^{r}(\bmod p)$ and $\tilde{Y}_{j} \equiv \tilde{Y} /\left(y_{j}\right)^{r}(\bmod p)$ respectively, and by taking $\bar{k} \equiv \bar{Y}^{-1}(\bmod p)$ as the key.

Consider a variant of the CWC system for which the chairperson $U_{0}$ sends $U_{j}$ only $c_{1}$ and $\tilde{Y}_{j}$, and not $c_{2}$ which, as observed earlier does not authenticate $U_{0}$ (in this case $U_{0}$ must be authenticated some other way - see Section 4.3). We shall show that cracking this variant by a passive eavesdropper (a 'ciphertext attack') is as hard as cracking the Diffie-Hellman [6] problem,

Input: $g, p, g^{a} \bmod p, g^{b} \bmod p ;$ Output: $g^{a b} \bmod p$.

Indeed suppose that it is easy to crack the modified key distribution system and let $g^{a} \bmod p, g^{b} \bmod p$ be an instance of the Diffie-Hellman problem. Set $c_{1}=g^{a} \bmod p, y_{0}=g^{b} \bmod p$ and $y_{i}=g^{t_{i}-b} \bmod p$, for $i=1,2, \ldots, n$, where the $t_{i} \in Z_{p-1}$ are chosen randomly. Then $g^{r} \equiv g^{a}(\bmod p)$ and $\vec{Y}_{j} \boxminus\left(g^{a}\right)^{T-t_{j}}(\bmod p)$, where $T=\sum_{i=1}^{n} t_{i}$. We are assuming that it is easy to compute the key,

$$
\tilde{k} \equiv \tilde{Y}^{-1} \equiv\left(g^{b}\right)^{a(1-n)} \cdot g^{a(n b-T)} \equiv g^{a b-a T}(\bmod p),
$$

so it is easy to compute $g^{a b} \equiv \tilde{k} \cdot\left(g^{a}\right)^{T}(\bmod p)$, and hence to find a solution for the Diffie-Hellman problem. The reduction in the reverse direction is straightforward: if it is easy to crack the Diffie-Hellman problem, then it is easy to compute $\tilde{k} \equiv\left(\left(y_{0}\right)^{n-1} \cdot \prod_{i=1}^{n} y_{i}\right)^{-r}(\bmod p)$, from $\left(y_{0}\right)^{n-1} \cdot \prod_{i=1}^{n} y_{i}(\bmod p)$ and $g^{-r} \bmod p$.

For this variant of the CWC system we also get some protection from known key attacks ('plaintext attacks') by active adversaries. This follows by observing that 'old-session' information: $c_{1}=g^{r} \bmod p, \bar{Y}_{j} \equiv\left(y_{0}{ }^{n-1} \cdot \prod_{i \neq j} y_{i}\right)^{r}(\bmod p)$, and $\bar{k} \equiv\left(y_{0}^{n-1} \cdot \prod_{i=1}^{n} y_{i}\right)^{r}(\bmod p)$, can be simulated, and that therefore the argument used in [8] for 'non-paradoxical' systems applies. However it should be pointed out that there is a flaw [5] in the proof given in [8], and consequently the proposed variant may not be 'proven secure' for known key attacks (in the general case).

\subsection{Using a composite modulus}

To prevent the attack in Section 2 we may also use a composite modulus $m=p q$, where $p, q$ are appropriate primes, and take $g$ to be an element of large order, e.g. a primitive element of $Z_{p}$ and $Z_{q}{ }^{1}$ Then it is not necessary to modify $Y$ and $Y_{j}$. For a 'provably secure' protocol we may use the variant in the previous section with composite modulus (however in this case the probability distributions are not uniform and we must use randomized reductions [2] as in [8]). Again we get some protection from known key attacks. 


\subsection{Addressing the authentication problem}

As pointed out in Section 3 the validator $c_{2}$ does not authenticate the chairperson $U_{0}$, since anybody can compute it without knowing the secret key $x_{0}$. To prevent this we may replace $c_{2}$ by $\tilde{c}_{2 j}=\left(y_{j}\right)^{\tilde{k} x_{0}} \bmod p$. Clearly it is hard to compute $\tilde{c}_{2 j}$ without knowing $\tilde{k}$ and either $x_{0}$ or $x_{j}$, provided the Diffie-Hellman problem is hard. Furthermore any $U_{j}$ can easily validate $\tilde{k}$, since $\tilde{c}_{2 j} \equiv\left(y_{0}\right)^{\tilde{k} x_{j}}(\bmod p)$. However this modification offers no protection against insider attacks. Indeed any $U_{i}$ can compute $\left(y_{j}\right)^{x_{0}} \boxminus\left(y_{0}\right)^{x_{j}}(\bmod p)$ from $\tilde{c}_{2 j}$ (obtained by eavesdropping) and from the key $\tilde{k}[7,1]$. Then, at any later time, $U_{i}$ can impersonate $U_{0}$, or forge any key $\tilde{k}$.

There seems to be no obvious way of solving the authentication problem without using a separate authentication system. The scheme in [4] addresses this problem and other more general issues.

Acknowledgement. The author wishes to thank Yvo Desmedt and Dieter Gollmann for many helpful discussions.

\section{References}

1. L. Adleman, K.M. Manders, and G.M. Miller. On taking roots in finite fields. Annual Symposium on Foundations of Computer Science, Vol. 18, 1977, pp. 175178.

2. S. Ben-David, B. Chor, O. Goldreich, M. Luby. On the theory of Average case Complexity. Proceedings of the twenty first annual ACM Symp. Theory of Computing, STOC, 1977, pp. 175-178.

3. Chin-Chen Chang, Tzong-Chen Wu, and C.P. Chen. The Design of a Conference Key Distribution System. Presented at Auscrypt 92, Gold Coast, Australia, December 13-16, 1992. To appear in: Advances in Cryptology, Lecture Notes in Computer Science, Springer-Verlag.

4. M. Burmester, Y. Desmedt. An Efficient and Secure Conference Key Distribution System. Manuscript, April 1993.

5. Y. Desmedt, M. Burmester. Towards practical 'proven secure' authenticated key distribution. To appear in, Proceedings of the 1st ACM Conference on Communications and Computing Security, Fairfax, Virginia, November 3-5, 1993.

6. W. Diffie and M. E. Hellman. New directions in cryptography. IEEE Trans. Inform. Theory, IT-22(6), 1976, pp. 644-654.

7. D. Shanks. Five number theoretic algorithms. Proc. Second Manitoba Conf. Numerical Mathematics, 1972, pp. 51-70.

8. Y. Yacobi. A Key Distribution Paradox. In Advances in Cryptology - Crypto '90, Proceeding (Lecture Notes in Computer Science \#537), 1991, A.J. Menezes and S.A. Vanstone, Eds, Springer-Verlag, pp. 268-273. 